\title{
Control of Electrical Appliances through Voice Commands
}

\author{
Arthi.J.E ${ }^{1}$, M.Jagadeeswari ${ }^{2}$ \\ ${ }^{I}$ (P.G Scholar, Power Electronics and Drives, Sri Ramakrishna Engineering College, India) \\ ${ }^{2}$ (Professor \& Head, Department of ECE (PG) - VLSI, Sri Ramakrishna Engineering College, India)
}

\begin{abstract}
Voice recognition is an expanding trend nowadays for automation. As speech is the preferred mode of operation for human being, this paper highlights the importance of human voice that activates electrical appliances at home in wireless environment. The user makes use of voice commands to perform certain actions such as switching the lights ON/OFF and regulating the speed of appliances. This system plays an important role for the elderly and physically disables people to control their appliances in intuitive and flexible way. The graphical user interface (GUI) system is developed using LabVIEW. LabVIEW is a highly productive development environment that interacts with real-world data for creating custom applications. So the method of control of electrical appliances is implemented using LabVIEW. With this voice recognition technique accuracy of more than $90 \%$ is achieved.
\end{abstract}

Keywords: Automation, Voice recognition, LabVIEW, ON/OFF control, Speed regulation.

\section{INTRODUCTION}

Controlling appliances is a main part in automation. In future, voice recognition technique will be the method for controlling appliances, computers and robots. There is a huge demand waiting for this technology to come into effect. Voice recognition is a standalone and very less expensive technique that may be used to control electrical appliances. Elderly population worldwide is increasing rapidly as a result of the increase of the average life probability of people is the trend shown by the demography of the world population. These Automation Systems not only benefit the employed population but it also helps the disabled and elderly population.

A set of voice commands are functionally equivalent to the set of switches which is used to control wheelchair [1].

Home appliances control using Bluetooth based remote control [2]. In this a remote control with a Keypad which is interface to a microcontroller and this is interfaced to Bluetooth module to provide wireless interface for the remote to communicate with the appliances control module.

A Voice-Input Voice-Output Communication Aid (VIVOCA) recognizes the disordered speech of the user and builds messages, which are converted into synthetic speech [3]. The speech is processed and recognized by a speech recognizer.Mean recognition accuracy is $67 \%$.

This design implementation involves wireless automation of home loads by giving voice commands through personal computer and fault identification which gives the exact status of the load [4].

Control of home appliances through voice commands with mobile application [5] in this a mobile application is developed that converts the user voice command into SMS and send through GSM network which increases the software complexity.

The implementation of voice controlled automation, in a wireless medium, based on Microsoft Speech recognition [6].

Wireless Home Automation System (WHAS) has been proposed in literature [7]-[10]. The home automation system is intended to control all electrical appliances in a home or office using voice commands with low-power RF Zigbee wireless communication modules.

Controlling home appliances through a microcomputer uses a PC and PC parallel port to control the appliances [8]. Two different approaches are used to control the home appliances; approaches are timer option and voice command.

Multiplatform control system for home automation using LabVIEW in this the coded signals are sent through the home's wiring to switches and outlets that are programmed to operate appliances and electronic devices in every part of the house [9].

\section{VOICE RECOGNITION TECHNIQUE - OVERVIEW}

Voice recognition is the technology by which sounds, phrases spoken by individuals are converted into electrical signals and these signals are changed into coding patterns to which meaning is allocated. This concept could more generally be called as sound recognition or speech recognition. Human voice is mainly focused here, because we most naturally and most frequently use our voices to communicate our thoughts to others in our 
immediate environments. The user would possibly gain the greatest feeling of immersion to use their voice as the most common form of communication. Principle of voice recognition is shown in the Fig.1.

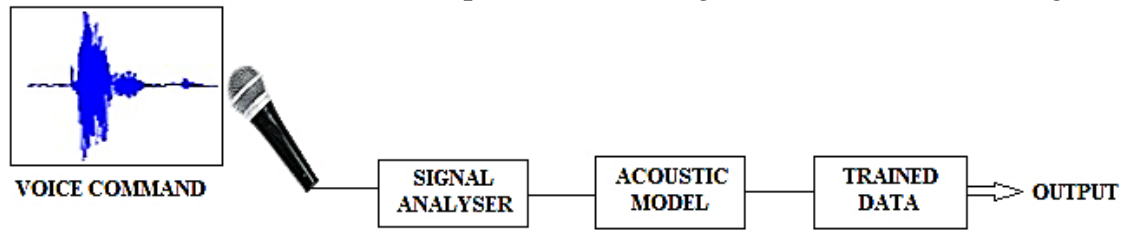

Fig.1. Principle of voice recognition

The first element that is required for voice recognition is the collection of speech data. Database of speech data is built from multiple speech samples then those samples of all the necessary speech data can be recorded and stored. The signal analyser passes the data from the speech sample to the acoustic model for identification. Samples of possible speech data is passed into language models. These possibilities are compared with previous results from the trained model. The speech data with the highest probability of a match is selected as being the correct data and given as output.

\section{PROPOSED SYSTEM DESCRIPTION}

An input voice is directly given to the microphone, which converts the voice command into electrical signal. The signal from the microphone is fed into voice recognition module. The general block diagram for the proposed method is shown in the Fig. 2 .

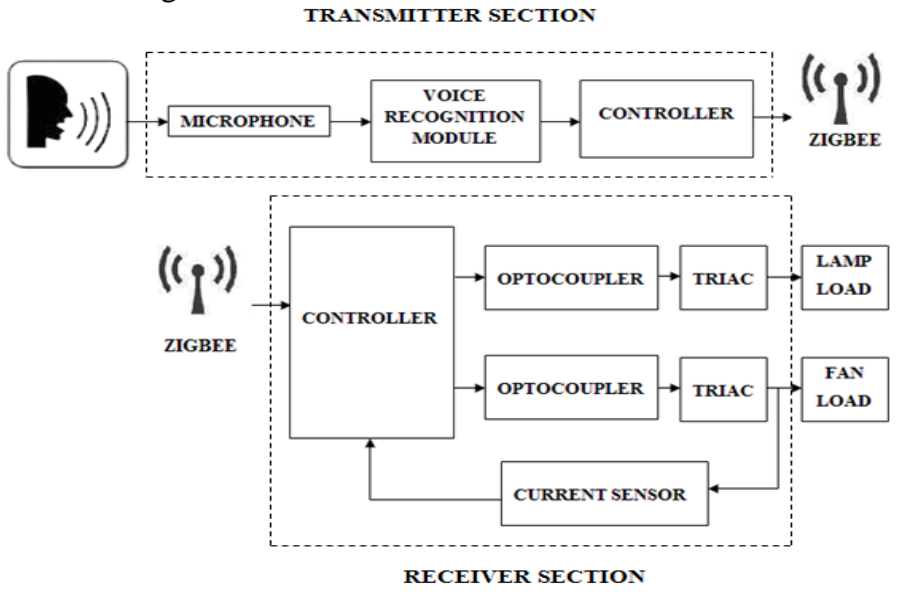

Fig.2. Block diagram to control appliances using voice recognition technique

The initial step in voice recognition is the person has to speak a word or phrase through microphone. Since each person's voice is different, new user's voice must be trained first in the program as an input before that user's voice can be recognized by the program. With the trained voice a statistical average of the multiple samples of the same word is analyzed by the program and stores the averaged sample as a template in a program data structure. When the averaged sample matches with the already stored sample then the corresponding signal is given to the controller. The device control program is stored in the controller memory to control the devices. From the controller the required signal is transmitted through zigbee. Upon receiving the signal, by program controlling the signal is given to TRIAC through an optocoupler driver circuit to drive the load.

Hidden Markov Model (HMM) algorithm is used for training. It is a statisticalMarkov model in which the system being modeled is a Markov process with unobserved (hidden) states. The observation is turned to be a probabilistic function (discrete or continuous) of a state instead of a one-to-one correspondence of a state [3]. Each state randomly generates one of M observations. Sequences of vectors of acoustic parameters are treated as observations of acoustic word models used to compute $\mathrm{P}\left(\mathrm{y}^{\mathrm{T}} \mid \mathrm{W}\right)$, the probability of observing a sequence $\mathrm{y}^{\mathrm{T}}$ of vectors where a word sequence $\mathrm{W}$ is pronounced. For a sequence $\mathrm{y}^{\mathrm{T}}$, a word sequence $\mathrm{W}$ is generated by the ASR system with a search process based on the rule is given in equation (1).

$$
\mathrm{W}=\arg \max \mathrm{P}\left(\mathrm{y}^{\mathrm{T}} \mid \mathrm{W}\right) \mathrm{P}(\mathrm{W})
$$

Where, $\mathrm{W}$ corresponds to the candidate having maximum probability. $\mathrm{P}\left(\mathrm{y}^{\mathrm{T}} \mid \mathrm{W}\right)$ is computed by Acoustic Models $(\mathrm{AM})$, while $\mathrm{P}(\mathrm{W})$ is computed by Language Models (LM). For more general voice recognition systems, a different output distribution is given to each word. A hidden Markov model for a sequence of words or phonemes is made by concatenating the individual trained hidden Markov models for the separate words and phonemes.

Appliances speed can be controlled with the help of Pulse-width modulation. The main reasons to prefer PWM for regulating motor speed are: 
$>$ It doesn't generate additional heat thus it is energy efficient.

$>$ It improves reliability because the fan doesn't run all the time at full speed.

$>$ It improves the acoustics of the load with high-frequency driving signals.

PWM is the method of applying a square wave signal to a load which will vary its speed by changing the duty cycle of the signal. PWM signal is used to control a switch that delivers the power needed to the load; it is not used to directly drive the load.

Four different duty cycles to control speed is shown in the Fig.3. Duty cycle is often used relating to electrical devices, e.g., switching power supplies. In an electrical device, a 70\% duty cycle corresponds to power ON period and 30\% corresponds to power OFF period of the time.

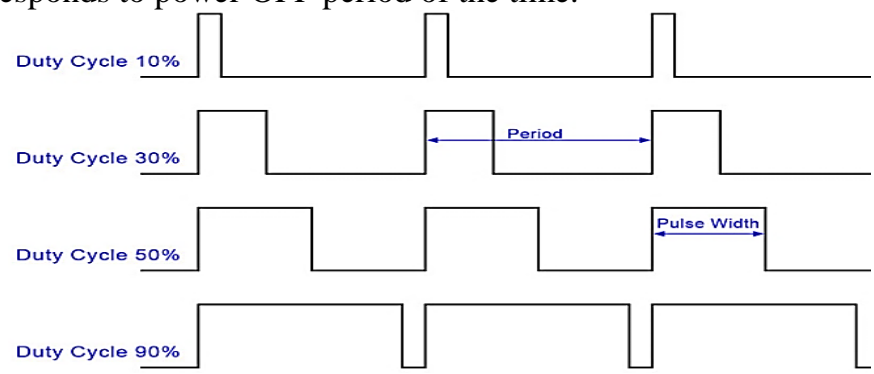

Fig.3. Four different duty cycles to control speed

$$
\text { Duty cycle }=\frac{\text { Pulse Width }}{\text { Period }} X 100
$$

A low duty cycle provides low speeds and high speeds are produced by high duty cycle. Full torque of the load can be used by controlling a load with PWM signal this is the main advantage of controlling a load with PWM instead of a real analog signal.

\section{EXPERIMENTAL SETUP IN LABVIEW}

The simulation diagram for switching lights ON/OFF is shown in the Fig.4. The words to be stored in speech initializer are given to the phrases. The action "Light ON" is executed when the spoken word from the microphone is matched with the already stored command in the speech initializer block.

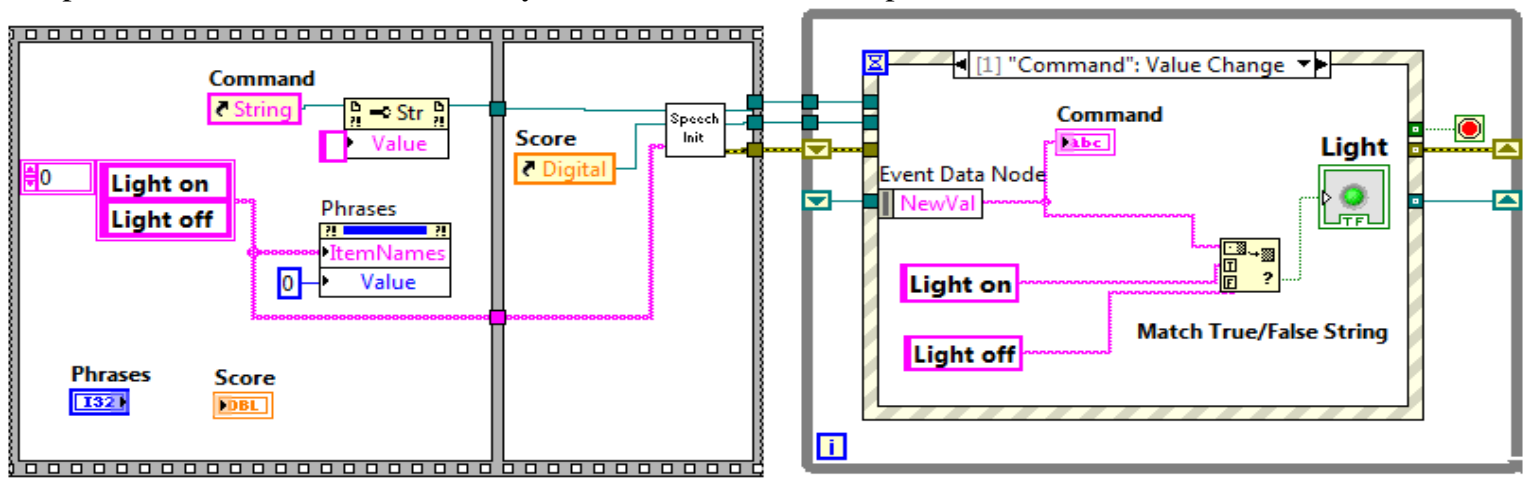

Fig.4. Simulation diagram for ON/OFF control

The diagram for speech initializer block is shown in the Fig. 5.

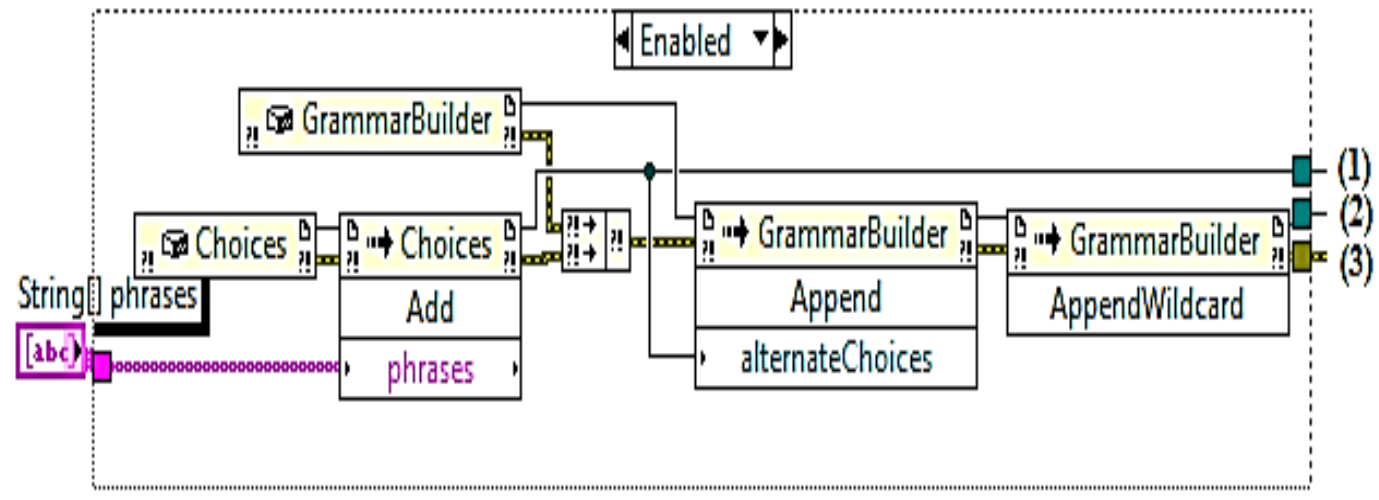




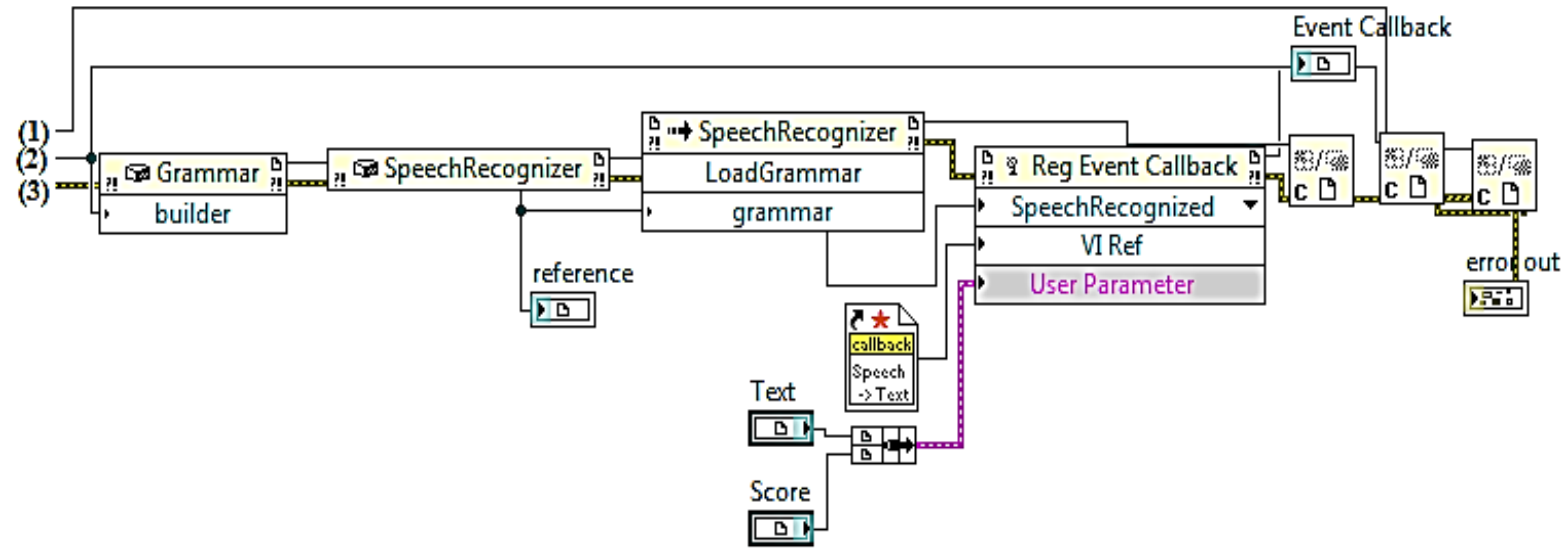

Fig.5. Simulation diagram for speech initializer block

In this the words from the string are given to the training process which is shown in the simulation diagram of speech initializer. When multiple samples of same word is recognized then that word is taken as template and it checks for the already stored word.

The simulation diagram for speed regulation is shown in the Fig.6.The speed control commands are given to the speech initializer block. The voice commands one, two and four are converted into word by speech initializer block and it compared with the words in the phrases.
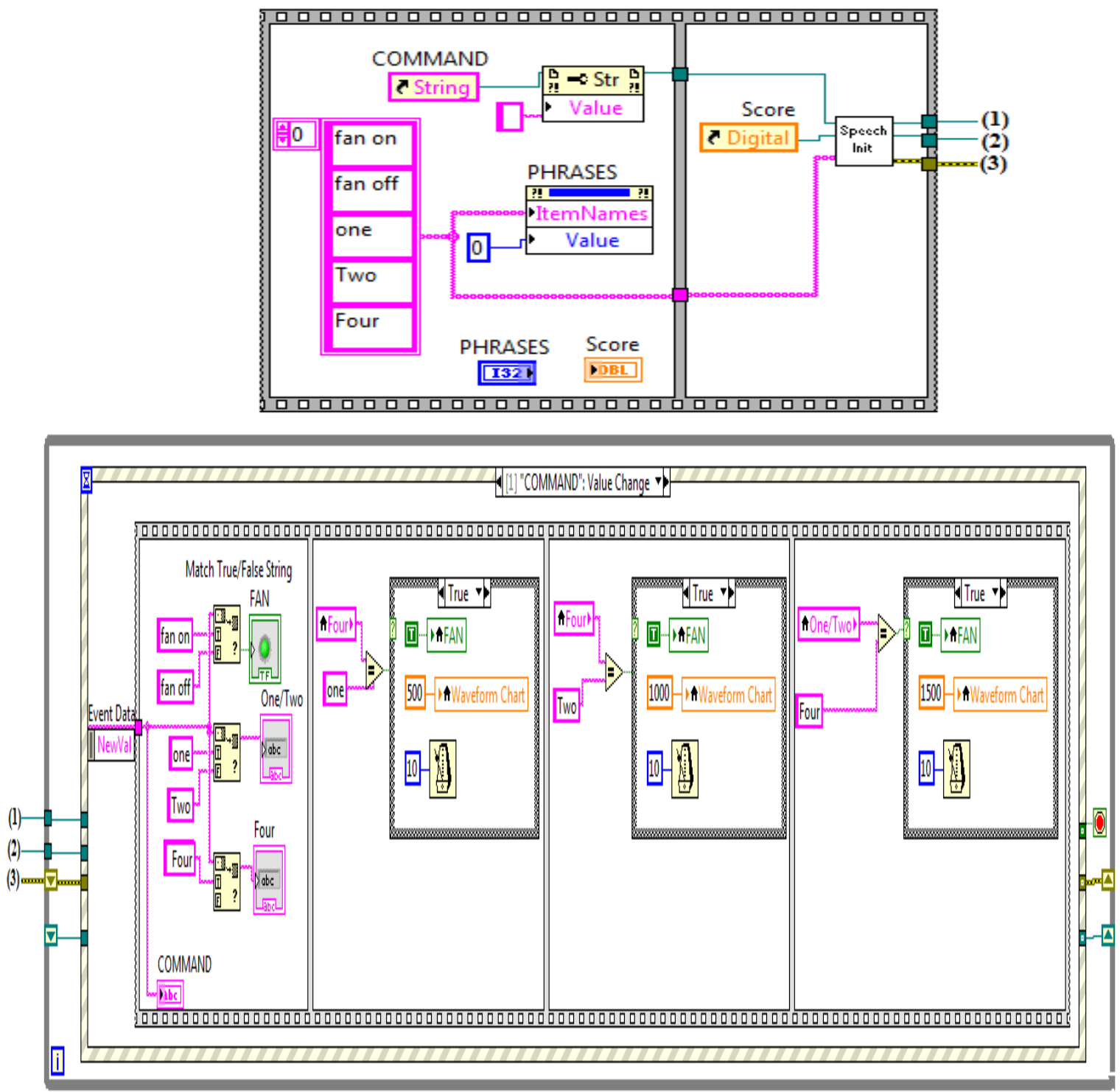

Fig.6. Simulation diagram for speed regulation 
a. On/off control

V. RESUlTS AND DiSCUSSIONS

Simulated output for ON/OFF control is shown in the Fig.7.

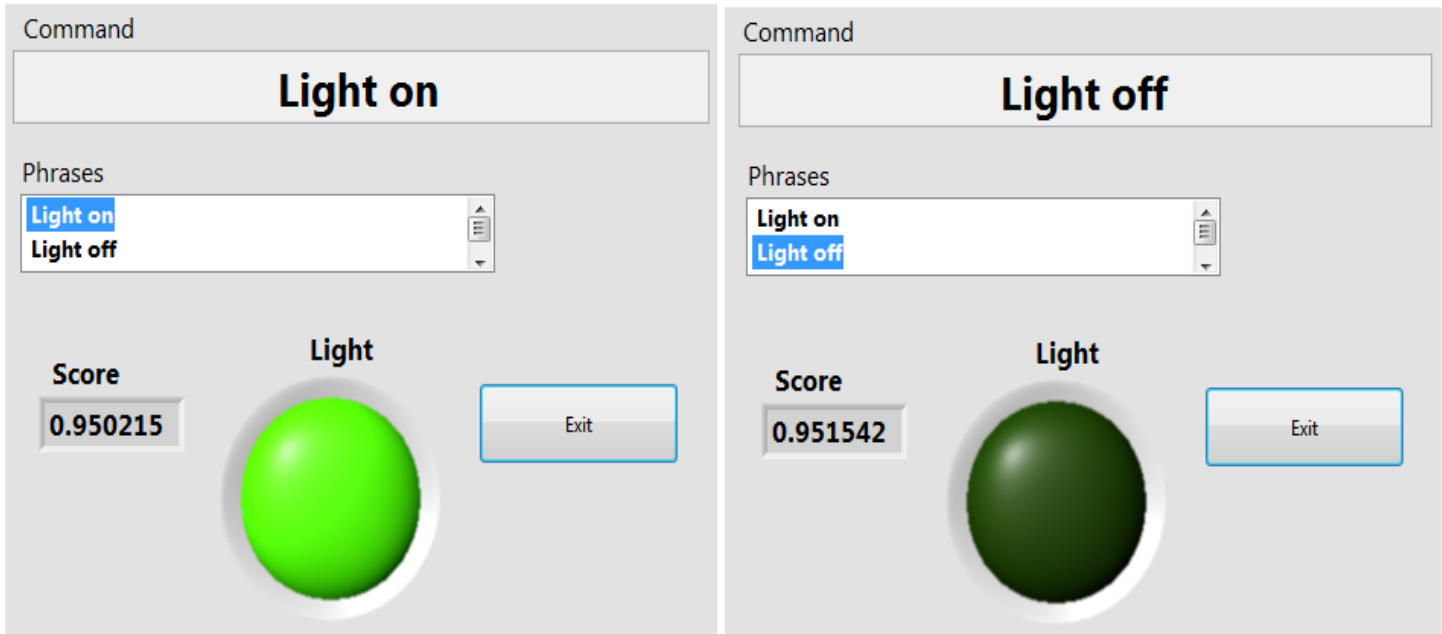

Fig.7. Output for ON/OFF control

For ON/OFF control, spoken word is displayed in command with the help of voice recognition technique and it compares with the already stored word in phrases, if it matches it makes the light to switch ON otherwise it won't respond. The value of the score gives the accuracy of each spoken words by voice recognition.

\section{b. Speed regulation}

In speed regulation, for the identified word PWM pulses are generated and the corresponding firing angle is given to the TRIAC and the voltage is given across the load for each spoken word 'ONE', 'TWO' and 'FOUR'. The simulated output for speed control is shown in the Fig.8.

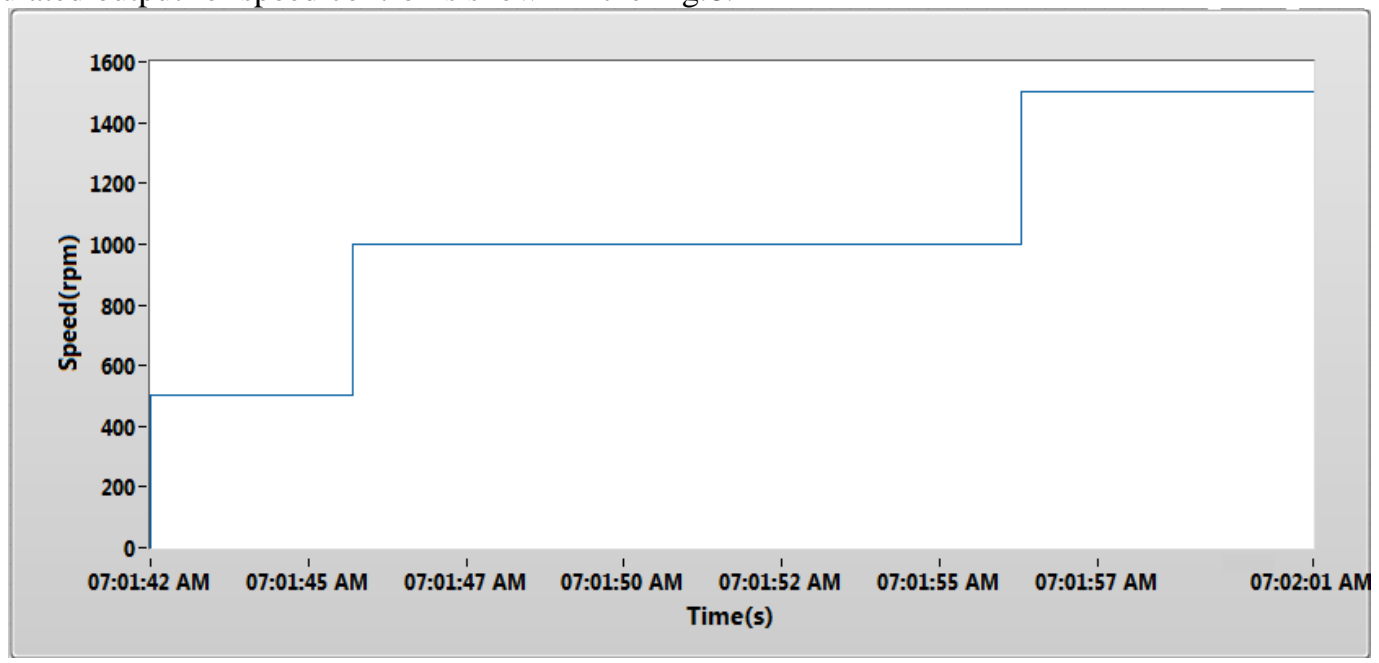

Fig.8. Output waveform for speed control by voice recognition technique

The commands and their respective speed in rpm are as follows: One $-500 \mathrm{rpm}$, Two - $1000 \mathrm{rpm}$ and Four $1500 \mathrm{rpm}$. The input command which is used for this result is one, two, four and the speed waveform is generated according to the command given as per the time shown in the system

\section{CONCLUSION}

Control of appliances using voice recognition technique is simulated using LabVIEW software and the results have been presented to demonstrate the proposed system. Initially, it is applied to switch lights ON/OFF, and then it is also extended to vary the speed of any appliances. The level of speed was found to vary for each commands and the speed level is displayed in the simulation. The simulated result shows that these loads were achieved with a voice recognition accuracy of $95 \%$. This system recognizes the input commands very well and manages to give a good response. This system can be applied in electrical appliances like fan, fridge, air conditioner, television, etc. 
In future work it is possible to make tests with a large data set of voice command and also the number of appliances to be controlled can be increased. In can also be fine-tuned with the help of neural networks to train the voice commands.

\section{REFERENCES}

[1] Richard.C, Simpson and Simon.P.Levine, "Voice Control of a Powered Wheelchair", IEEE Trans. Neural systems and Rehabilitation Engineering, vol. 10, no. 2, pp. 122-125, June 2002.

[2] Laisa.C.P, Nichholas S. Almeida, Ana G. D. Correa, Roseli D.Lopes, Marcelo K.Zuffo, "Accessible Display Design to Control Home Area Networks", IEEE Trans. Consumer Electronics, Vol.59, No.2, pp. 422-427, 2013.

[3] Mark S. Hawley, Stuart P. Cunningham, Phil D. Green, Pam Enderby, Rebecca Palmer, Siddharth Sehgal, and Peter O’Neill, “A Voice-Input Voice-Output Communication Aid for People with Severe Speech Impairment”, IEEE Trans. Neural systems and Rehabilitation engineering, vol. 21, no. 1, pp. 23-31, , Jan 2013.

[4] Gnanasekar. A.K, Jayavelu.P, Nagarajan.V, "Speech Recognition Based Wireless Automation of Home Loads with Fault Identification", IEEE International conference on communications and signal processing (ICCSP), Vol. 3, pp.128-132, 2012.

[5] Faisal Baig, Saira Beg and Muhammad Fahad Khan, "Zigbee Based Home Appliances Controlling Through Spoken Commands Using Handheld Devices", International Journal of Smart Home Vol. 7, No. 1, pp. 19-26, Jan 2013.

[6] Jeyasree Tagore, 1.Gayathri, Kamin Uttamambigai.S.P, "Implementation of Wireless Automation of Home Loads Based on Microsoft SDK", International Journal of Science and Technology, Vol. 2, No. 5, pp.402-404, 2013.

[7] Y.Usha Devi, "Wireless Home Automation System Using Zigbee", International Journal of Scientific \& Engineering Research, Vol. 3, pp. 1-5, 2012.

[8] S. M. Anamul Haque, S. M. Kamruzzaman and Md. Ashraful Islam, "A System for Smart-Home Control of Appliances Based on Timer and Speech Interaction", Proceedings of the 4th International Conference on Electrical Engineering \& 2nd Annual Paper Meet, Vol.2, pp. 128-131, Jan 2006.

[9] Basil Hamed, "Design \& Implementation of Smart House Control Using LabVIEW", International Journal of Soft Computing and Engineering (IJSCE), Vol. 1, Issue-6, pp. 98-106, Jan 2012.

[10] I.K. Hwang, D.S. Lee and J.W. Baek, "Home Network Configuring Scheme for All Electric Appliances Using ZigBee-based Integrated Remote Controller”, IEEE Trans. Consumer Electronics, vol. 55, No. 3, pp. 1300-1307, August 2009. 